\title{
Malária autóctone no Município de Rio Branco, Estado do Acre, Brasil, no período de 2003 a 2010
}

\author{
Autochthonous malaria in the Municipality of Rio Branco, Acre State, Brazil, from 2003 to 2010
}

Malaria autóctona en el Municipio de Rio Branco, Estado de Acre, Brasil, en el período de 2003 a 2010

Igor Gomes dos Santos

Curso de Medicina, Universidade Federal do Acre, Rio Branco, Acre, Brasil
Rita do Socorro Uchôa da Silva

Centro de Ciências da Saúde e do Desporto, Universidade Federal do Acre, Rio Branco, Acre, Brasil

\begin{abstract}
RESUMO
A malária ocorre com maior frequência nos municípios do interior da Região Amazônica, sendo notificados casos autóctones da doença em algumas capitais brasileiras. Este trabalho foi realizado objetivando descrever a incidência e a distribuição de casos autóctones de malária no Município de Rio Branco, capital do Estado do Acre. Trata-se de estudo retrospectivo, do tipo série histórica. Foram notificados 5.394 casos autóctones da doença em Rio Branco no período de 2003 a 2010 . Houve predomínio de: infecção por Plasmodium vivax (3.713 casos, 73,9\% do total); acometimento do sexo masculino (3.268 casos, 65, $1 \%$ do total); e faixa etária de 10 a 19 anos (24,3\% do total). As localidades rurais foram responsáveis por $3.252(60,3 \%)$ casos, sendo o Projeto de Assentamento Dirigido Padre Peixoto (Linha 3 Gleba Z I) a área mais atingida pela doença. Na região urbana, os bairros Taquari e Centro ( $1^{\circ}$ Distrito) foram os mais acometidos. Concluiu-se pela existência de uma tendência decrescente na incidência dos casos autóctones de malária em Rio Branco, além de uma mudança do perfil da infecção, com redução de casos originários das localidades rurais, que foram suplantados pelos ocorridos em localidades periurbanas.
\end{abstract}

Palavras-chave: Malária; Vigilância Epidemiológica; Plasmodium vivax.

\section{INTRODUÇÃO}

A malária é uma doença infectoparasitária responsável por alta morbimortalidade no mundo. Estudos estimam que mais de $40 \%$ da população mundial está exposta ao risco de adquirir a doença'.

Considerada um dos maiores problemas de saúde pública no Brasil, a malária concentra-se em áreas endêmicas favorecidas pelo clima tropical. A Região Amazônica é a área de maior incidência da doença, o que torna a ocorrência da malária no Brasil quase exclusiva desta região, com registro de $99,8 \%$ do número total de casos. Condições socioeconômicas e ambientais favorecem a proliferação dos vetores do Plasmodium, tornando elevada a exposição dos seus habitantes ao agente etiológico da malária ${ }^{2}$. Somando-se a isto, dados sugerem que a elevada incidência da doença nesta região está relacionada também à expansão urbana desordenada e sem planejamento, fatos comuns nessa área ${ }^{3}$.

\footnotetext{
Correspondência / Correspondence / Correspondencia: Igor Gomes dos Santos

Rua das Acácias, 648. Bairro: Jardim Tropical

CEP: 69901-233 Rio Branco-Acre-Brasil

Tel.: (68) 9964-1313

E-mail: igorgs@msn.com
}

Nos Estados da Amazônia Legal não há registros uniformes de casos de malária, ocorrendo diferentes graus de risco de contrair a doença dentro da região, os quais são classificados de acordo com a Incidência Parasitária Anual (IPA) ${ }^{4}$.

O IPA é definido como o índice que expressa o número de casos por 1.000 habitantes. Por meio deste índice se pode estimar o risco de adoecer por malária em determinado local e período. Classificam-se as áreas como de alto, médio e baixo risco de transmissão (maior que 49,9/1.000 habitantes: alto risco; entre $10 \mathrm{e}$ 49,9/1.000 habitantes: médio risco; de 0,1 a 9,9/1.000 habitantes: baixo risco e IPA igual a zero: áreas sem risco de transmissão de malária)'.

Várias capitais da Região Amazônica registram casos autóctones de malária. Em Manaus, Estado do Amazonas, a incidência da doença é considerada epidêmica devido à presença de um alto número de migrantes que, ao abandonarem suas roças e se deslocarem para a periferia da cidade, favoreceram a multiplicação de criadouros de anofelinos, instalando, assim, o ciclo de transmissão ${ }^{5}$.

Entre os anos de 2001 e 2005, foram registrados, nas zonas urbana e rural de Manaus, 211.313 casos autóctones de malária. A maioria dos casos (141.180 $66,8 \%)$ ocorreu na área urbana, sendo $70.133(33,2 \%)$ casos na área rural ${ }^{5}$. 
Tanto áreas periurbanas de Manaus, como de Porto Velho, Estado de Rondônia, são consideradas epidêmicas para a malária em função do constante afluxo de população, que, em busca de melhores oportunidades de emprego, se desloca das áreas de assentamento agrícola para áreas urbanas. Acrescente-se a isto a instalação de favelas, que favorece a multiplicação dos criadouros ${ }^{6}$.

Dados do Sistema de Informação de Vigilância Epidemiológica - Malária (Sivep-Malária) mostraram que, dos 296.056 casos autóctones registrados no Amazonas nos anos de 2003 e 2004, 125.246 (42,3\%) eram autóctones de Manaus?.

Pará figura entre os Estados da Amazônia brasileira com maior morbidade por malária, registrando, respectivamente, nos anos de 2001 e 2002, 48\% e 53,5\% da totalidade de casos registrados na Amazônia Legal ${ }^{8}$. Belém, a capital do Estado, notificou 650 casos de malária autóctone no ano de 2004 e 360 casos em 2005, sendo todos causados pelo $P$. vivax, principalmente nas localidades de Cotijuba e Bonfim?"

Estado do Tocantins, apesar de pertencer à Região Amazônica, é atualmente considerado como área não endêmica, pela baixa incidência de malária, porém ainda com risco de transmissão da doença. Dos casos registrados nos municípios de Tocantins, no período de 1998 a 2006, Palmas registrou 4,2\% de autoctonia ${ }^{10}$.

No Estado do Acre, a exploração da borracha no final do século XIX contribuiu para um aumento significativo na incidência da malária, devido à elevada imigração de nordestinos para a região, sem exposição prévia à doença ${ }^{11}$. Após esta fase, fatores relacionados à imigração de pessoas das áreas rurais para as urbanas, com ocupação urbana não planejada e o clima da região têm sido referidos como causas da manutenção da ocorrência da doença ${ }^{12}$.

Durante o ano de 2006, o Estado do Acre registrou uma epidemia de malária, atingindo 93.863 casos registrados da doença (a imensa maioria proveniente da região do Vale do Juruá). Neste ano, Cruzeiro do Sul - o maior centro urbano do Vale do Juruá - registrou IPA de $571,5 / 1.000$ habitantes, caracterizando a área como hiperendêmica ${ }^{12}$.

IPA registrado no Acre em 2007 foi de 68,6; uma redução de 47,3\% em relação ao IPA registrado em 2006. Mesmo com este decréscimo no número de casos, o Acre ainda figurou, em 2007, como o Estado com maior IPA entre os da Amazônia Legal ${ }^{13}$.

Em 2007, dos 79 municípios da Região Amazônica classificados como de alto risco para malária, três municípios acreanos que compõem o Vale do Juruá (Rodrigues Alves, Mâncio Lima e Cruzeiro do Sul) apresentaram incidência acima de 300/1.000 habitantes; sendo considerados prioritários pelo Plano Nacional de Controle da Malária (PNCM)'.

Este trabalho foi realizado visando conhecer a realidade dos casos autóctones de malária no Município de Rio Branco, para alertar tanto as autoridades quanto os profissionais de saúde que atuam no município.

\section{MATERIAIS E MÉTODOS}

Este estudo é retrospectivo, do tipo série histórica, sobre os casos autóctones de malária originários de Rio Branco, e notificados no período de janeiro de 2003 a dezembro de 2010 .

Em Rio Branco, capital do Estado do Acre, está quase metade da população residente no território acreano, totalizando 336.038 habitantes $\left(38,03 \mathrm{hab} . / \mathrm{km}^{2}\right)$. Rio Branco é o centro administrativo, econômico e cultural da região Vale do Acre. Limita-se com os municípios de Bujari e Porto Acre, juntamente com o Estado do Amazonas ao norte; os municípios de Xapuri e Capixaba, ao sul; Senador Guiomard, a leste; e Sena Madureira, a oeste ${ }^{14}$.

Para a realização deste trabalho, os dados coletados foram obtidos nos arquivos da Secretaria Municipal de Saúde de Rio Branco/Departamento de Vigilância Epidemiológica/Seção de Controle de Endemias/ Programa de Controle de Malária, registrados no SivepMalária da Fundação Nacional de Saúde (Funasa)/ Secretaria de Vigilância em Saúde (SVS) do Ministério da Saúde (MS).

Sivep-Malária é um sistema de informação para notificação dos casos de malária diagnosticados no Brasil, que foi implantado em 2003 e que funciona a partir da inclusão, em um banco de dados, das informações contidas nas fichas de notificação de malária, sendo veiculados por meio da Internet, permitindo acesso direto dos órgãos municipais, estaduais e federais.

As variáveis avaliadas no estudo foram: faixa etária; gênero; índice parasitário anual; espécies de plasmódios infectantes; parasitemia; bairros e localidades de Rio Branco incriminados como locais prováveis da infecção malárica.

A estratificação das zonas urbana, periurbana e rural foi feita baseada na classificação das localidades registradas no Sivep-Malária. Os bairros e conjuntos foram considerados área urbana. Vilas e colônias foram agrupadas como área periurbana e, por último, seringais, fazendas, ramais e projetos de assentamento dirigidos foram considerados como zona rural.

Há grandes limitações na utilização dos dados do Sivep. Uma delas é a existência de inúmeras localidades não registradas em nenhum outro sistema de informações, mas que subsidiam o registro de casos da doença no referido sistema.

trabalho em questão analisou dados secundários, não havendo implicações éticas, pois os mesmos foram obtidos mediante termo em que os autores se comprometeram em preservar a privacidade dos sujeitos da pesquisa. $\bigcirc$ protocolo de pesquisa foi submetido ao Comitê de Ética do Hospital das Clínicas do Acre/FUNDHACRE e aprovado sob o parecer número 631/2011, em 4 de agosto de 2011. 


\section{RESULTADOS}

No Município de Rio Branco, foram notificados 5.394 casos autóctones de malária no período de 2003 a 2010 , sendo que em $374(6,9 \%)$ destes não havia registro da localidade provável de infecção malárica. A frequência média anual foi de 674 casos da doença, com variação de 148 (em 2009) a 1.582 casos (em 2004).

O gráfico 1 evidencia o número de casos autóctones registrados nos anos de 2003 a 2010 em Rio Branco, apresentando, também, a linha de tendência dos casos registrados. Observou-se um pico no número de casos da doença durante o ano de 2004, com notificação de 1.582 casos de malária autóctone, havendo sucessiva redução nos anos subsequentes, porém com pequeno aumento em 2010, quando se registraram 199 casos.

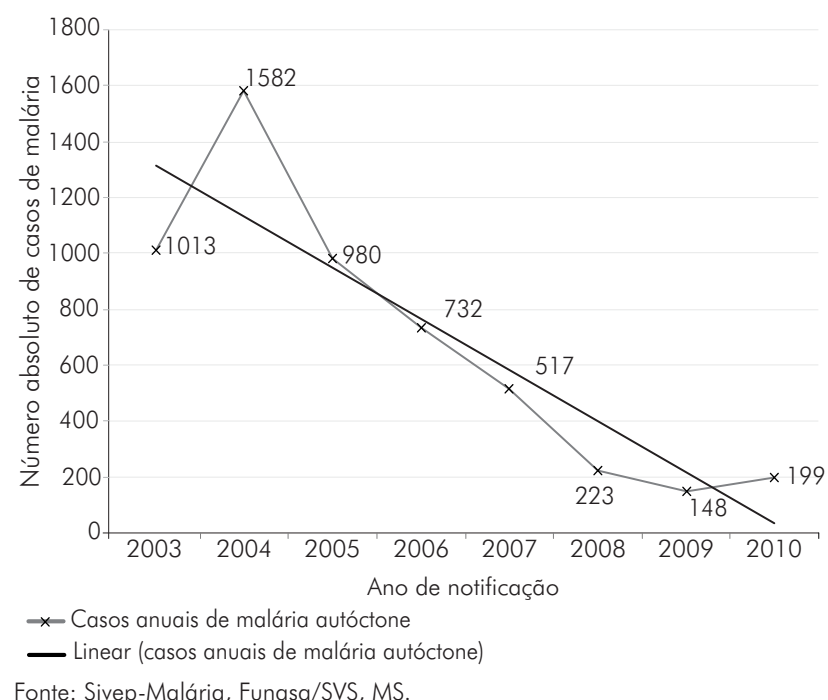

Gráfico 1 - Total de casos positivos de malária considerados autóctones com tendência linear, Município de Rio Branco, Acre, 2003 a 2010
O gráfico 2 ilustra a distribuição temporal dos casos, com nítido predomínio de casos de malária pelo $P$. vivax. Mostra uma tendência decrescente, tanto nos casos de malária pelo $P$. vivax como pelo $P$. falciparum.

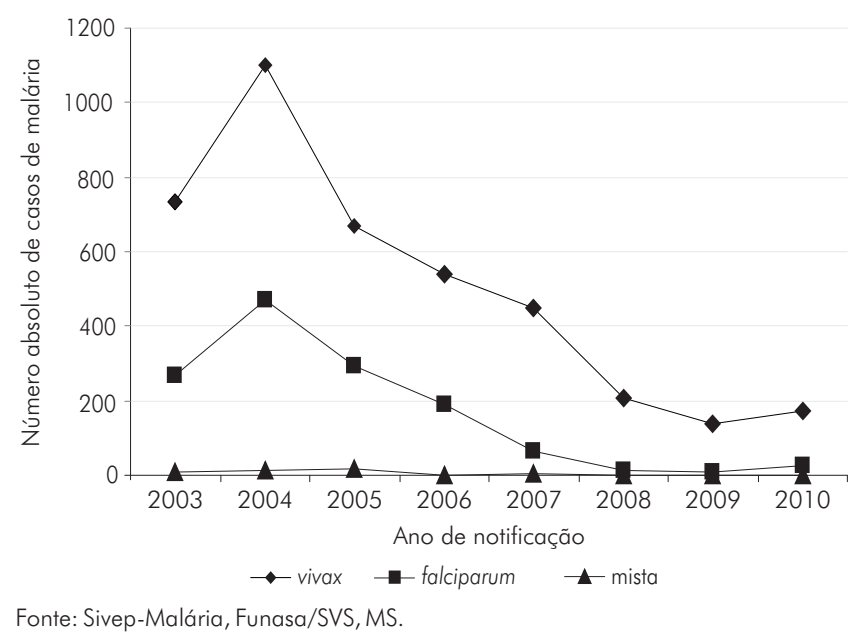

Gráfico 2 - Número de exames parasitológicos (gota espessa ou esfregaço) positivos de acordo com espécie de Plasmodium detectada em casos de malária considerados autóctones do Município de Rio Branco, Acre, 2003 a 2010

A maioria dos casos $(65,1 \%)$ de malária autóctone em Rio Branco ocorreu em indivíduos do sexo masculino, sendo o $P$. vivax responsável por 3.713 $(73,9 \%)$ das infecções, seguido pelo $P$. falciparum com 1.265 casos $(25,2 \%)$ e a infecção mista $(P$. vivax e $P$. falciparum) em 42 casos $(0,84 \%)$ conforme mostra a tabela 1 . Em toda série histórica não foram notificados casos de malária porP. malariae ou P. ovale.

Tabela 1 - Casos de malária autóctone notificados em Rio Branco, Estado do Acre, segundo gênero, espécie de Plasmodium e IPA, 2003 a 2010

\begin{tabular}{|c|c|c|c|c|c|c|c|c|c|c|c|c|}
\hline \multirow{3}{*}{ Ano } & \multirow{2}{*}{\multicolumn{2}{|c|}{ Homens }} & \multirow{2}{*}{\multicolumn{2}{|c|}{ Mulheres }} & \multirow{2}{*}{\multicolumn{6}{|c|}{$\begin{array}{l}\text { Espécies de Plasmodium } \\
\text { P. vivax }\end{array}$}} & \multirow{3}{*}{ IPA } & \multirow{3}{*}{$\begin{array}{c}\text { Total de } \\
\text { casos }\end{array}$} \\
\hline & & & & & P. falciparum & & & & & & & \\
\hline & $\mathrm{N}$ & $\%$ & $\mathrm{~N}$ & $\%$ & $\mathrm{~N}$ & $\%$ & $\mathrm{~N}$ & $\%$ & $\mathrm{~N}$ & $\%$ & & \\
\hline 2003 & 637 & 66,63 & 319 & 33,37 & 255 & 26,67 & 695 & 72,70 & 6 & 0,63 & 10,9 & 956 \\
\hline 2004 & 996 & 64,93 & 538 & 35,07 & 458 & 29,86 & 1.065 & 69,43 & 11 & 0,71 & 11,8 & 1.534 \\
\hline 2005 & 560 & 60,87 & 360 & 39,13 & 286 & 31,09 & 617 & 67,06 & 17 & 1,85 & 8,9 & 920 \\
\hline 2006 & 444 & 66,37 & 225 & 33,63 & 174 & 26,01 & 494 & 73,84 & 1 & 0,15 & 6,4 & 669 \\
\hline 2007 & 316 & 66,53 & 159 & 33,47 & 53 & 11,16 & 419 & 88,21 & 3 & 0,63 & 6,7 & 475 \\
\hline 2008 & 128 & 67,02 & 63 & 32,98 & 11 & 5,76 & 179 & 93,72 & 1 & 0,52 & 4,4 & 191 \\
\hline 2009 & 75 & 63,56 & 43 & 36,44 & 8 & 6,78 & 109 & 92,37 & 1 & 0,85 & 4,3 & 118 \\
\hline 2010 & 114 & 71,25 & 46 & 28,75 & 20 & 12,50 & 138 & 86,25 & 2 & 1,25 & 3,2 & 160 \\
\hline Total $^{\dagger}$ & 3.268 & 65,10 & 1.752 & 34,90 & 1.265 & 25,20 & 3.713 & 73,96 & 42 & 0,84 & - & 5.020 \\
\hline
\end{tabular}

Fonte: Sivep-Malária, Funasa/SVS, MS.

* Malária mista (F: falciparum + V: vivax).

${ }^{\dagger}$ Casos de malária com localidades não informadas foram excluídos desta tabela.

Sinal convencional utilizado: - Dado numérico igual a zero não resultante de arredondamento. 
A faixa etária com o maior número de casos de malária autóctone em Rio Branco foi de 10 a 19 anos (1.017; $24,35 \%)$, seguindo-se a de 20 a 29 anos (938; 22,46\%) e a de 0 a 9 anos $(850 ; 20,35 \%)$, totalizando os três grupos etários $2.805(67,16 \%)$ casos. Dentre os casos registrados, 361 indivíduos com malária (6,7\%) residiam na área urbana de Rio Branco, 1.409 (26,12\%) na região periurbana e $3.252(60,3 \%)$ na região rural.

Na região urbana de Rio Branco, as localidades com maior número de casos foram: Taquari (61 casos), Centro no $1^{\circ}$ Distrito ( 25 casos) e Vila Acre ( 18 casos). Na região periurbana destacaram-se as seguintes localidades:
Barro Alto (237 casos), Caipora (155 casos) e Santa Maria (122 casos), enquanto que na região rural destacaram-se as localidades Projeto de Assentamento Dirigido-PAD Padre Peixoto Linha 3 Gleba Z I (345 casos), Colônia Paraná Linha 4 - rua da Palheira (301 casos) e PAD Padre Peixoto Linha 2 Gleba Z I (288 casos).

A tabela 2 apresenta a distribuição dos casos de malária autóctone de Rio Branco, de acordo com as localidades mais afetadas, agrupadas por região urbana, periurbana e rural. As localidades que mais registraram casos de malária foram responsáveis por 2.055 casos (38\%) registrados no período de 2003 a 2010.

Tabela 2 - Distribuição do número de casos autóctones de malária em Rio Branco, Estado Acre, segundo os prováveis locais de infecção (localidades), 2003 a 2010

\begin{tabular}{|c|c|c|c|c|c|c|c|c|c|c|c|}
\hline & \multirow{2}{*}{ Localidade* } & \multicolumn{8}{|c|}{ Ano } & \multicolumn{2}{|c|}{ Total $^{\dagger}$} \\
\hline & & 2003 & 2004 & 2005 & 2006 & 2007 & 2008 & 2009 & 2010 & $\mathrm{~N}$ & $\%$ \\
\hline \multirow{5}{*}{ 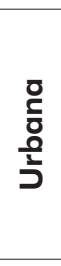 } & Taquari & 6 & 12 & 4 & 30 & 4 & 1 & - & 4 & 61 & 1,1 \\
\hline & Centro ( $1^{\circ}$ Distrito) & 2 & 4 & 8 & 2 & 3 & 2 & 2 & 2 & 25 & 0,5 \\
\hline & Vila Acre & 1 & 2 & 1 & 1 & 2 & 4 & 2 & 5 & 18 & 0,3 \\
\hline & Cidade Nova & 2 & 3 & 3 & 2 & 1 & 1 & - & 1 & 13 & 0,2 \\
\hline & Bosque & 5 & 1 & 1 & 1 & - & - & 1 & 1 & 10 & 0,2 \\
\hline \multirow{5}{*}{ 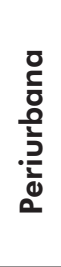 } & Barro Alto & 13 & 100 & 25 & 50 & 12 & 13 & 11 & 13 & 237 & 4,4 \\
\hline & Caipora & 14 & 69 & 47 & 19 & 4 & 1 & - & 1 & 155 & 3,0 \\
\hline & Santa Maria & 8 & 6 & 25 & 33 & 26 & 8 & 8 & 8 & 122 & 2,2 \\
\hline & Santa Cecília & 2 & 4 & 6 & 41 & 26 & 2 & - & 11 & 92 & 1,7 \\
\hline & Belo Jardim II & 6 & 18 & 12 & 26 & 14 & 4 & 2 & 1 & 83 & 1,5 \\
\hline \multirow{5}{*}{$\overline{\overline{0}}$} & $\begin{array}{l}\text { PAD Padre Peixoto (Linha } \\
3 \text { Gleba Z I) }\end{array}$ & 106 & 161 & 63 & 15 & - & - & - & - & 345 & 6,4 \\
\hline & $\begin{array}{l}\text { Colônia Paraná (Linha } 04 \\
\text { - R. da Palheira) }\end{array}$ & 11 & - & 94 & 42 & 51 & 49 & 18 & 36 & 301 & 5,6 \\
\hline & $\begin{array}{l}\text { PAD Padre Peixoto (Linha } \\
2 \text { Gleba Z I) }\end{array}$ & 90 & 129 & 64 & 5 & - & - & - & - & 288 & 5,3 \\
\hline & $\begin{array}{l}\text { PAD Padre Peixoto (Linha } \\
1 \text { Gleba Z II) }\end{array}$ & 49 & 82 & 30 & 3 & - & - & - & - & 164 & 3,0 \\
\hline & $\begin{array}{l}\text { Seringal São Francisco do } \\
\text { Espalha }\end{array}$ & 9 & 30 & 69 & 20 & 13 & - & - & - & 141 & 2,6 \\
\hline
\end{tabular}

Fonte: Sivep-Malária, Funasa/SVS, MS.

* Foram expressas na tabela as localidades que mais registraram casos nos oito anos, excluídas as demais localidades.

† Porcentagem em relação ao número total de casos notificados no período de 2003 a 2010.

Sinal convencional utilizado: - Dado numérico igual a zero não resultante de arredondamento.

No Município de Rio Branco foram registrados 13.181 casos de malária (autóctones e importados) no período de 2003 a 2010, o que correspondeu a 3,9\% dos casos registrados em todo o Estado do Acre no mesmo período (338.623 casos).

Dos exames positivos para malária em Rio Branco, durante os oito anos referentes a este estudo (13.181), 40,9\% foram autóctones (5.394 casos), correspondendo a 1,9\% dos casos autóctones no território estadual durante o período estudado.

\section{DISCUSSÃO}

Apesar de serem conhecidas as limitações dos estudos que usam bancos de dados secundários, estes são importantes para avaliar e aprimorar as informações usadas nos sistemas de vigilância. Sabe-se que tais dados são muitas vezes coletados, porém não são devidamente analisados, o que poderia ser útil para fins de políticas públicas e intensificação de ações de controle e prevenção em nível local ${ }^{15}$.

Em toda série histórica houve registro de infecção autóctone de malária dentro do Município de Rio Branco, sendo a maior parte dos casos notificados provenientes das localidades rurais do município, fato já esperado em virtude das condições ambientais normalmente propícias à transmissão da doença nessas áreas.

Um estudo realizado em Manaus, entre 2001 e 2005, sobre os casos autóctones de malária nas zonas urbana e 
rural, detectou que $66,8 \%$ dos casos ocorreram na área urbana e $33,2 \%$ na rural e revelou que as zonas Leste, Norte e Oeste da cidade foram os locais que mais registraram casos $(99,4 \%$ do total de casos da área urbana), apontando tais dados como consequência de desmatamentos e da expansão acentuada da área urbana de Manaus ${ }^{7,15}$. Diferentemente do que foi detectado em Manaus, o presente estudo identificou que a maior proporção de casos autóctones de malária em Rio Branco foi proveniente de localidades da zona rural (60,3\% dos casos) no período de 2003 a 2010 . Talvez a diferença de metodologia tenha contribuído para esta diferença entre os dois estudos, pois em Rio Branco foi incluída a classificação de áreas periurbanas, enquanto que em Manaus a estratificação não foi continha tal parâmetro.

A diferença citada anteriormente ocorreu quando se comparou os números absolutos dos dois estudos, porém, quando comparado o IPA entre as três zonas agrupadas nesta pesquisa (urbana, periurbana e rural), verifica-se que os moradores da região rural apresentaram maior risco de adquirir a doença, visto que, a média do IPA no período estudado (2003 a 2010) foi de $51,8 / 1.000$ habitantes (considerado alto risco), enquanto a zona periurbana apresentou IPA de $22,4 / 1.000$ habitantes (médio risco) e a zona urbana, por sua vez, 0,6/1 .000 habitantes (baixo risco).

Quanto à espécie de Plasmodium, o presente estudo evidenciou resultados semelhantes a outros realizados, havendo predomínio de $P$. vivax como agente da infecção, o qual foi o responsável por $73,9 \%$ dos casos autóctones notificados em oito anos (2003 a 2010). Em outras capitais da Região Amazônica como Manaus ${ }^{6}$ e Belém 9 , estudos mostraram predominância da espécie de $P$. vivax, refletindo uma característica regional e até nacional, visto que a maioria dos casos registrados ocorre por esta espécie ${ }^{16}$.

À semelhança de outros estudos, em Rio Branco o gênero com maior número de casos de malária autóctone registrados foi o masculino $(65,1 \%)$, provavelmente pela maior exposição dessas pessoas ao vetor, devido suas atividades laborais $^{14}$.

É sabido que a partir do ano de 2005 o Acre apresentou um aumento nos casos, com registros de 57.242 mil casos, culminando, no ano de 2006, para o registro histórico de 93.863 casos de malária no Estado. Com diversas medidas usadas para o controle intensivo da malária, o número de casos da doença, que foi de 51.294 no ano de 2007, reduziu-se em 2008 para 27.730 casos. A capital, Rio Branco, não mostrou qualquer alteração na reta descendente que vinha apresentado referente ao número de casos registrados da doença. Isto só reforça a impressão de que a epidemia registrada foi consequente à elevada incidência na região do Estado do Acre denominada de Vale do Juruá, composta pelos municípios de Cruzeiro do Sul, Mâncio Lima, Rodrigues Alves, Porto Walter e Marechal Thaumaturgo, os quais, em conjunto, foram responsáveis por $87,2 \%$ dos casos registrados no Estado em $2007^{12}$.

Tal epidemia ocorrida no Acre entre 2005 e 2008, provavelmente foi influenciada pela escavação de açudes para piscicultura, atividade esta subsidiada pelo Governo do Estado do Acre nessa região a partir do ano de $2005^{12}$.

Rio Branco figura como localidade de baixo risco para infecção por malária, estando sua IPA sempre abaixo de 6/1.000 habitantes durante a série histórica descrita neste trabalho. Registrou no ano de 2004 o máximo de 5,5 casos/1.000 habitantes e em 2010 o menor índice $(0,7 \text { casos } / 1.000 \text { habitantes })^{17}$. Esta baixa incidência parasitária é uma característica da região do Baixo Acre, a qual Rio Branco integra. Tal região, apesar de registrar um número absoluto elevado (comparado a outras regiões, como as do Alto Acre e Purus) possui, entretanto, a mais alta concentração populacional do Estado, o que explica a incidência parasitária anual em baixos níveis.

Como acontece em toda a Amazônia, onde a ocorrência da malária não é uniforme, fica demonstrado que Rio Branco não se constitui em uma localidade de alta incidência da doença, diferente de Manaus, capital do Estado do Amazonas, localizado ao norte do Estado do Acre, que apresentou IPA de 45,4 casos/1.000 habitantes no ano de 2003 (médio risco) ${ }^{7}$.

A redução do número de casos de malária em Rio Branco, demonstrada pela tendência linear de queda ao longo desta série histórica, provavelmente pode estar associada às medidas intensificadas de combate aos vetores, ao trabalho de vigilância epidemiológica constante, ao melhor diagnóstico, com implantação de laboratórios estrategicamente instalados, à educação em saúde nas localidades com registro de casos e às melhores condições de trabalho da equipe de combate às endemias.

combate estratégico ao vetor, realizado pelos órgãos responsáveis pelo controle de endemias nas localidades com registro de infecção por malária, em Rio Branco, pode ser um dos prováveis motivos da redução dos casos de malária registrados no município, a ponto de, nos anos de 2009 e 2010, ter sido registrado um número superior de casos autóctones nas áreas periurbanas sobre as zonas rurais (sem aumento dos casos periurbanos, mas diminuição dos casos nas localidades rurais), como foi visto neste estudo.

É conhecido o fluxo ocupacional para estes espaços periurbanos, principalmente por uma população proveniente da área rural em busca de emprego nas áreas urbanas, criando a instalação de favelas, novas áreas habitacionais, conjuntos habitacionais e invasões, locais caracteristicamente desprovidos de infraestrutura, resultado de um processo desorganizado e recente de urbanização, o que mantém estas áreas com risco de transmissão da doença ${ }^{7,9}$.

No presente estudo, constatou-se que as localidades periurbanas que apresentaram maior ocorrência de 
casos, como Barro Alto, Caipora, Santa Maria, Santa Cecília e Belo Jardim possuem localização afastada do centro da cidade, processo de ocupação recente e carência de infraestrutura sanitária. Mas podem ser citados também bairros integrados à área urbana e próximos ao centro comercial da cidade que apresentaram registro de casos autóctones, como o bairro Taquari, que registrou, no período de 2003 e 2010, a maior proporção de casos dentro da zona urbana, e o bairro Cidade Nova, que registrou o quarto maior número de casos em uma localidade urbana. Ambos têm alta densidade populacional, ocupação desordenada e invasões. Soma-se a isto o fato destas duas localidades citadas serem atingidos pelas cheias do rio Acre, o que repercute no aumento da incidência de doenças infectoparasitárias.

Quanto à completude das variáveis, neste estudo observou-se perda de informação por não preenchimento, ou desconhecimento, na variável localidades, com registro de 6,9\% de localidades não informadas. Já a variável idade apresentou a maior proporção de perdas, com supressão de dados em 22,6\% dos casos, sendo que os anos de 2009 e 2010 foram os que apresentaram maior perda proporcional, com $48 \%$ e $53 \%$ respectivamente. Estas perdas refletem uma real necessidade de aprimorar o preenchimento da ficha de notificação obrigatória, que alimenta diretamente o Sistema de Informação.

Mesmo com sua implantação recente, a análise do SIVEP-Malária deixa claro a má qualidade na coleta de informações de malária. Dados de maior qualidade podem fornecer uma abordagem mais objetiva na execução de políticas públicas locais.

\section{CONCLUSÃO}

A malária autóctone no Município de Rio Branco vem apresentando uma tendência decrescente, porém ainda está presente em pequeno número de casos em determinadas localidades e, apesar de considerada controlada pelo serviço de vigilância epidemiológica, exige constante vigilância para evitar que a infecção se torne presente no meio urbano, como ocorre em Cruzeiro do Sul, segundo maior centro populacional do Estado do Acre, onde há elevados índices de transmissão malárica no meio urbano.

Com uma tendência decrescente dos casos de malária em Rio Branco no período registrado no estudo, de 2003 a 2010, a infecção por malária provavelmente caminhará para um controle pontual dos casos notificados, desde que haja manutenção das ações realizadas pelo órgão de controle de endemias, como ações de controle químico e bloqueio espacial, sobretudo na zona rural, e vigilância de novos casos no perímetro periurbano.

Logo, este estudo expôs uma realidade que parecia ser remota, porém bastante real e presente no Município de Rio Branco: a autoctonia de casos de malária. Autoctonia esta não apenas proveniente de zonas rurais da cidade, mas presente em localidades periurbanas e urbanas. Alerta-se para uma situação que, mesmo possuindo atual status de infecção "controlada" pelos órgãos governamentais, a malária autóctone em Rio Branco pode vir a se tornar um problema de maiores proporções, caso medidas não sejam tomadas para reduzir sua ocorrência.

Estudo como este possui grande importância para alertar os médicos e demais profissionais de assistência à saúde quanto à possibilidade da existência de malária autóctone no Município de Rio Branco. Portanto, mesmo apresentando baixo índice, a malária deve ser considerada nas hipóteses diagnósticas de síndromes febris agudas, mesmo que não haja relatos de viagens ou deslocamentos de Rio Branco.

\section{Autochthonous malaria in the Municipality of Rio Branco, Acre State, Brazil, from 2003 to 2010} ABSTRACT

Malaria occurs more frequently in the interior municipalities of the Amazon Region, and autochthonous cases of the disease have been reported in some Brazilian capitals. The present study was conducted to describe the incidence and distribution of autochthonous malaria cases in the Municipality of Rio Branco, the capital of the Brazilian State of Acre. This is a retrospective study based on a historic series. A total of 5,394 cases of autochthonous malaria were reported in Rio Branco in the period from 2003 to 2010 . There was a predominance of Plasmodium vivax infections $(3,713$ cases, $73.9 \%$ of the total), with more frequent infections being observed in men (3,268 cases, $65.1 \%$ of the total) and the 10 - to 19 -year age group (24.3\% of the total). Rural areas accounted for 3,252 (60.3\%) cases, with the Projeto de Assentamento Dirigido Padre Peixoto (Linha 3 Gleba Z I) being the most affected area. Among the urban areas, Taquari and Centro (1 st District) were the most affected districts. It was concluded that there was a decreasing trend in the incidence of autochthonous malaria cases in Rio Branco. There was also a change in the infection profile, with a reduction in cases originating in rural areas, which were replaced by more cases in peri-urban localities.

Keywords: Malaria; Epidemiological Surveillance; Plasmodium vivax. 


\section{Malaria autóctona en el Municipio de Rio Branco, Estado de Acre, Brasil, en el período de 2003 a 2010 \\ RESUMEN}

La malaria ocurre con más frecuencia en los municipios del interior de la Región Amazónica, notificándose casos autóctonos de la enfermedad en algunas capitales brasileñas. Este trabajo fue realizado con el objetivo de describir la incidencia y la distribución de casos autóctonos de malaria en el Municipio de Rio Branco, capital del Estado de Acre, Brasil. Se trata de un estudio retrospectivo, de tipo serie histórica. Fueron notificados 5.394 casos autóctonos de la enfermedad en Rio Branco en el período de 2003 a 2010. Hubo predominio de: infección por Plasmodium vivax (3.713 casos, 73,9\% del total); acometimiento del sexo masculino (3.268 casos, 65,1\% del total); y franja etaria de 10 a 19 años (24,3\% del total). Las localidades rurales fueron responsables por 3.252 (60,3\%) casos, y el Projeto de Assentamento Dirigido Padre Peixoto (Linha 3 Gleba Z I) fue el área más atacada por la enfermedad. En la región urbana, los barrios Taquari y Centro ( $1^{\circ}$ Distrito) fueron los más acometidos. Se concluye por la existencia de una tendencia decreciente en la incidencia de los casos autóctonos de malaria en Rio Branco, además de un cambio en el perfil de la infección, con reducción de casos originarios de las localidades rurales, que fueron suplantados por los ocurridos en localidades peri-urbanas.

Palabras clave: Malaria; Vigilancia Epidemiológica; Plasmodium vivax.

\section{REFERÊNCIAS}

1 Ministério da Saúde (BR). Secretaria de Vigilância em Saúde. Guia de Vigilância Epidemiológica. 7. ed. Brasília: Ministério da Saúde; 2010. p.31-54. (Série Normas e Manuais Técnicos).

2 Oliveira-Ferreira J, Lacerda MVG, Brasil P, Ladislau JLB, Tauil PL, Daniel-Ribeiro CT. Malaria in Brazil: an overview. Malar J. 2010 Apr;9:115.

3 Almeida LB, Barbosa MGV, Martinez-Espinosa FE. Malária em mulheres de idade de 10 a 49 anos, segundo o SIVEP-Malária, Manaus, Amazonas, 20032006. Rev Soc Bras Med Trop. 2010 maiojun;43(3):304-8.

4 Ministério da Saúde (BR). Secretaria de Vigilância em Saúde. Situação Epidemiológica da Malária no Brasil. Brasília: Ministério da Saúde; 2008.

5 Saraiva MGG, Amorim RDS, Moura MAS, MartinezEspinosa FE, Barbosa MGV. Expansão urbana e distribuição espacial da malária no Município de Manaus, Estado do Amazonas. Rev Soc Bras Med Trop. 2009 set-out; 42(5):515-22.

6 Barata RCB. Malária no Brasil: panorama epidemiológico na última década. Cad Saude Publica. 1995 jan-mar; 11 (1):128-36.

7 Saraiva MGG. Malária em Manaus: análise epidemiológica, conhecimento e atitudes da população [Dissertação]. Manaus: Universidade Federal do Amazonas; 2007.

8 Santos RLC, Sucupira IMC, Lacerda RNL, Fayal AS, Póvoa MM. Inquérito entomológico e infectividade durante epidemia de malária no Município de Anajás, Estado do Pará. Rev Soc Bras Med Trop. 2005 marabr;38(2):202-4

9 Renault CS, Bastos FA, Filgueira JPPS, Homma TK. Epidemiologia da malária no Município de Belém Pará. Rev Para Med. 2007 jul-set;21 (3): 19-24.
10 Gener MES. Características epidemiológicas da malária autóctone no Estado do Tocantins, Brasil, 1998 a 2006 [Dissertação]. Brasília: Universidade de Brasília; 2008.

11 Cesário M, Cesário RR. Malária, Amazônia e desenvolvimento. Sci Am Brasil. 2006;54:54-5.

12 Costa KMM, Almeida WAF, Magalhães IB, Montoya R, Moura MS, Lacerda MVG. Malária em Cruzeiro do Sul (Amazônia Ocidental brasileira): análise da série histórica de 1998 a 2008. Rev Panam Salud Publica. 2010 nov;28(5):353-60.

13 Ministério da Saúde (BR). Secretaria de Vigilância em Saúde. Sistema de Informação de Vigilância Epidemiológica. Notificação de casos de malaria: relatório. Brasília: Ministério da Saúde; 2008.

14 Couto RD, Latorre MRDO, Santi SM, Natal D. Malária autóctone notificada no Estado de São Paulo: aspectos clínicos e epidemiológicos de 1980 a 2007. Rev Soc Bras Med Trop. 2010 jan-fev; 43(1):52-8.

15 Marques AC. Situação Epidemiológica da Malária no Brasil. In: $2^{\circ}$ Congresso Brasileiro de Epidemiologia; 1992; Belo Horizonte. Belo Horizonte: Associação Brasileira de Saúde Coletiva; 1992.

16 Ministério da Saúde. Secretaria de Atenção à Saúde. Vigilância em saúde: Dengue, Esquistossomose, Hanseníase, Malária, Tracoma e Tuberculose. 2. ed. Brasília: Ministério da Saúde; 2008.

17 Ministério da Saúde. Secretaria de Vigilância em Saúde. Sistema Nacional de Vigilância em Saúde: relatório de situação: Acre. 5. ed. Brasília: Ministério daSaúde; 2011.

Recebido em / Received / Recibido en: 28/4/2012 Aceito em / Accepted / Aceito en: 9/8/2012 